\title{
Performance of Low-Mass and High Thermal Conductivity Hybrid for High Track Density Environment
}

\author{
Y. Ikegami, A. Clark, D. Ferrere, S. Gonzalez-Sevilla, K. Hara, T. Kohriki, S. Terada, Y. Unno, and M. Weber
}

\begin{abstract}
We have fabricated a low-mass and high thermal conductivity hybrid for high track density environment, such as the super LHC experiment. The characteristics of the front-end chip were evaluated with connecting microstrip sensors of different lengths to the ASIC inputs.
\end{abstract}

\section{INTRODUCTION}

$\mathrm{R}$ ECENT silicon microstrip detectors are designed to incorporate VLSI chips on the detector to readout the signals and communicate with the outside data acquisition system. The hybrid, the base for the VLSI chips and related circuit components, is required to be low mass to reduce multiple coulomb scattering effects for precision tracking. The proposed silicon strip detector at the super LHC experiment includes a short strip silicon detector system in order to cope with the high track density. The system has about 10,000,000 readout channels. In order to minimize the number of hybrids, a hybrid reads out 2 segments of strips with two rows of readout chips; one row in each edge. Thus, the density of readout chips is quite high, 20 chips on a hybrid in this application. We designed and fabricated a new hybrid, applying a technology of copper-polyimide flexible circuit board and carbon-carbon reinforce material which have been developed and adopted for the present LHC ATLAS silicon strip detector (SCT [1]). This report is focused on the characteristics of the front-end chip on the hybrid with connecting microstrip sensors of different lengths to the ASIC inputs.

\section{ModulE DESIGN}

Fig. 1 shows a module design for the barrel SCT upgrade toward the super LHC experiment. It is a double side module, which can give $3 \mathrm{D}$ space points. The double side module is made of an upper and a lower single side sensor and a central baseboard and 4 separate hybrids. These hybrids are bridging over the sensor area with air-gap. A direct wire bonding

Manuscript received November 12, 2009

The research was partly supported by Grant-in-Aid for Scientific Research (A) (Grant No. 20244038, 40151956) and on Priority Area (Grant No. 20025007), in Japan.

Y. Ikegami (e-mail: ikegami@post.kek.jp), T. Kohriki, S. Terada and Y. Unno are with the IPNS, High Energy Accelerator Research organization; KEK, Tsukuba, Ibaraki 305-0801, Japan.

A. Clark, D. Ferrere, S. Gonzalez-Sevilla and M. Weber are with the Section de physique, Université de Genève, 24, rue Ernest Ansermet CH-1211 Genève, Switzerland.

K. Hara is with the Institute of Pure and Applied Sciences, University of Tsukuba, Tsukuba, Ibaraki 305-8571, Japan. scheme is adopted between front end chips and sensors, in order to obtain higher-density. The maximum fanning angle amounts to 16 degrees.

\section{A. Sensor}

The n-in-p type strip sensors [2] have been developed to cope with a fluence up to $10^{16} 1-\mathrm{MeV} \mathrm{n} \mathrm{n}_{\mathrm{eq}} / \mathrm{cm}^{2}$. The sensor size is $97.5 \mathrm{~mm} \times 97.5 \mathrm{~mm}$. The sensor has 4 segmentations for the requirement from hit occupancy. Each strip length is about $24 \mathrm{~mm}$. Each segment has 1,280 strips, corresponding to $74.5 \mu \mathrm{m}$ pitch.

\section{B. Baseboard}

The baseboard provides mechanical, electrical and thermal support. There are two types of baseboard substrates: thermalized pyrolytic graphite and carbon-carbon. Its thickness is $300 \mu \mathrm{m}$. A parylene coating technique is adopted to prevent delimitation of the substrate. Four AlNa facings are attached to the substrate. They have precision holes for the module mounting. The thermal performance of the module is discussed in [3].

\section{Hybrid}

Each hybrid has 20 front-end readout ASICs (ABCNext [4]), which can readout 128 channels. One module has 80 readout $\mathrm{ABCNext}$ chips 10,240 readout channels. The hybrid provides the full specifications operation of ABCNext chips, including the functions such as a redundancy operation and various powering and grounding schemes.

The flexible circuit size is $136 \mathrm{~mm} \times 28 \mathrm{~mm}$. The starting core for build-up is a double-sided $\mathrm{Cu}$ /polyimide $(\mathrm{PI})$ sheet in the middle. A single-sided $\mathrm{Cu} / \mathrm{PI}$ sheet is glued on each side of the core sheet. The thickness of $\mathrm{Cu}$ layer and PI base film is $12 \mu \mathrm{m}$ and $25 \mu \mathrm{m}$, respectively. In the cable part, the top and bottom copper layers are removed. The total thickness of hybrid part and of cable part is $0.260 \mathrm{~mm}$ and $0.165 \mathrm{~mm}$, respectively. Electrical connections among different layers are realized by either through-holes, penetrating all layers, or laser-cut via-holes between two adjacent layers. All $\mathrm{Cu} / \mathrm{PI}$ sheets are made with adhesive-less technology. The total weight of bare flexible circuit considerably decreased to $1.91 \mathrm{~g}$ from $3.09 \mathrm{~g}$ by button plating, which is also called pads-only plating or spot plating. The layer 1 and layer 2 include the main circuit patterns for ASICs with redundancy lines. The layer 3 and layer 4 are mainly for the power distribution and grounding, respectively. The detail description of flexible circuit is found in the reference [5]. 
The hybrid backed with a $400 \mu \mathrm{m}$ thick and $112 \mathrm{~mm}$ wide carbon-carbon sheet is designed to bridge over the silicon sensor avoiding any interference to the sensor surface. The carbon-carbon bridge having large heat conductivity of $670 \mathrm{~W} / \mathrm{m} / \mathrm{K}$ transfers the generated heat to the heat sink located at the legs of the bridge. The total weight (excluding electrical components) is $4.25 \mathrm{~g}$, having $0.00425 \mathrm{X} 0$ equivalent radiation lengths.

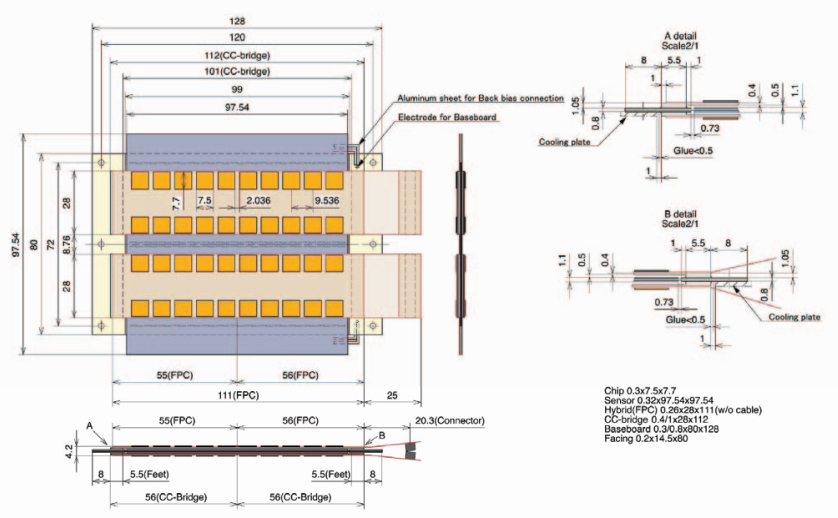

Fig. 1. Module design.

\section{Electrical Test Results}

The characteristics of the ABCNext ASIC were evaluated with connecting microstrip sensors of different lengths to the ASIC inputs. Fig. 2 shows a photo of the test module. The readout hybrid was mounted at the sensor end. We made the sensor, which had four different strip lengths, with wire bonding on the sensor. Two chips were stuffed for the longest strips $(=10 \mathrm{~cm})$ and one chip was stuffed for each of other lengths $(2.5,5.07 .5 \mathrm{~cm})$.

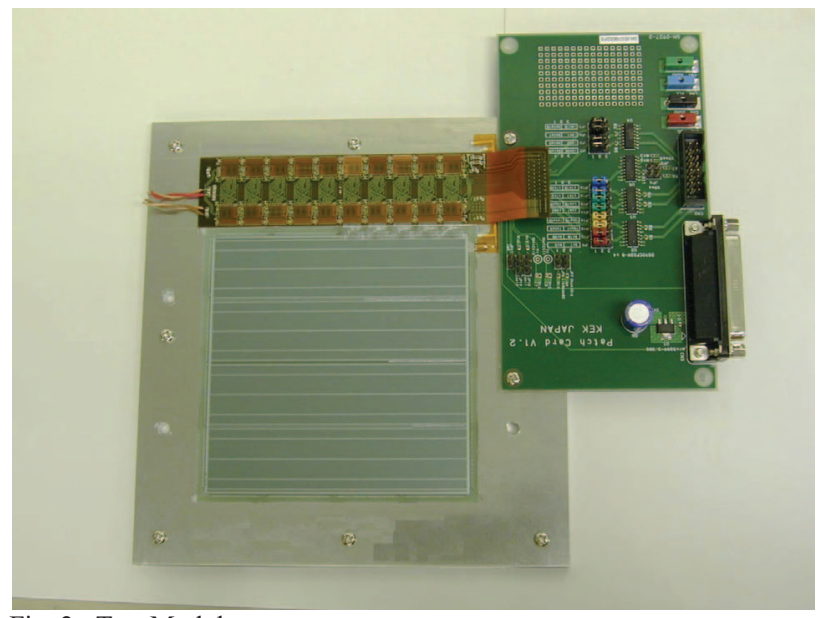

Fig. 2. Test Module.

Threshold scans were performed for injected charges of 1.5, 2.0 and $2.5 \mathrm{fC}$ with the internal calibration circuit mounted in the ABCNext chip. In each case a complementary error function was fitted to the data: the mean corresponds to the threshold at which 50\% efficiency was achieved for pulses of the designated magnitude and the sigma was a measure of the output noise (in $\mathrm{mV}$ ). The gain of each channel was calculated from a linear fit to the fit results for the three scans. The output noise from the scan taken with $2.0 \mathrm{fC}$ injected charge was divided by the gain to determine the input noise (in $\mathrm{fC}$ or ENC: Equivalent Noise Charge). The hybrid was kept at $15^{\circ} \mathrm{C}$ in a climate chamber. We also measured ENC with connecting external capacitances [6] instead of sensors. Two ENC measurements for the ABCNext were in agreement. Excess of ENC in the larger input capacitance region was observed. The reason is the increase of the parallel noise, since the bias resistance of the sensor became small with wire bonding on the sensor.

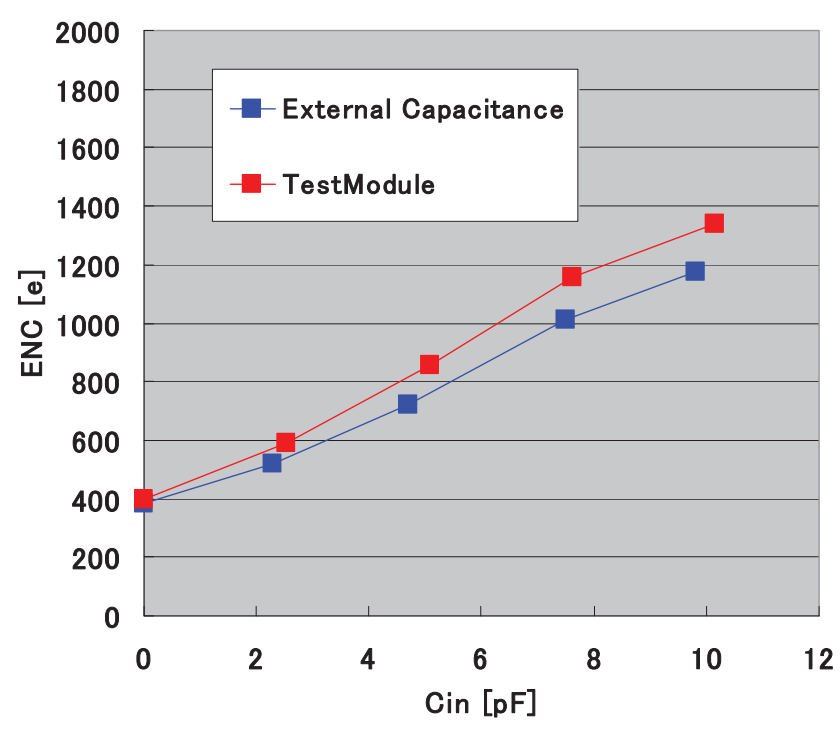

Fig. 3. ENC results.

\section{SUMMARY}

We have fabricated the low-mass, high-density, hybrid which provides the full functionality of the ABCNext full chips. The characteristics of the front-end chip were evaluated with connecting microstrip sensors of different lengths to the ASIC inputs. Two ENC measurements with connecting sensor and external capacitances were in agreement. The short strip module production is planned to start soon. 


\section{REFERENCES}

[1] ATLAS SCT Collaboration (A. Abdesselam et al.), The integration and engineering of the ATLAS SemiConductor Tracker barrel, Journal of Instrumentation (JINST), 3 (2008) P10006.

[2] K. Hara, et al., Development of Radiation Hard N+-on-P Silicon Microstrip Sensors for Super LHC, IEEE Trans. Nucl. Sci. 56 (2009) 468-473.

[3] T. Kohriki et al., Thermal Runaway Characteristics of Silicon Microstrip Module Designed for ATLAS Upgrade Inner Tracker at Super LHC, IEEE Nuclear Science Symposium CR N30-136, Dresden, Germany, October 19-25, 2008.

[4] J. Kaplon et al., The ABCN front-end chip for ATLAS Inner Detector Upgrade, Proceedings of Topical Workshop on Electronics for Particle Physics, TWEPP-08, Naxos, Greece, 15-19 Sep, 2008, CERN-20080008 (2008) 116.

[5] Y. Ikegami et al., Development of low-mass, high-density, hybrid for silicon microstrip sensors in very high radiation environment, to be published in proceedings of 7th International "Hiroshima" Symposium on Development and Applications of Semiconductor Tracking Devices.

[6] M. Mikeštíková et al., Testing of large area n-in-p silicon sensors intended for a very high radiation environment, to be published in proceedings of 7th International "Hiroshima" Symposium on Development and Applications of Semiconductor Tracking Devices. 